\title{
Nuevos retos para la lexicografía pedagógica bilingüe alemán-español
}

\author{
Meike Meliss \\ Universidad de Santiago de Compostela \\ meike.meliss@usc.es \\ Paloma Sánchez Hernández \\ Universidad Complutense de Madrid \\ palomash@filol.ucm.es
}

Resumenः El análisis de las obras lexicográficas existentes en español y en alemán para L2 confirma la necesidad de desarrollar un diccionario pedagógico con una nueva concepción, desde la cual el usuario pueda obtener una mayor información adaptada a sus necesidades. De especial relevancia es el tratamiento de la información específica con el que se puedan mejorar los procesos de producción de textos en L2 mediante un procedimiento que, teniendo en cuenta la diversidad de uso, permita seleccionar un lexema particular de la variedad de posibles lexicalizaciones.

En esta contribución se presentan los aspectos teóricos y metodológicos que sustentan el proyecto DICONALE-online. Los cuatro pilares del proyecto en torno al tipo de usuarios, al enfoque conceptual y onomasiológico, a la base empírica de los datos y al modelo de descripción enlazado con un punto de vista contrastivo configuran nuevos retos para el desarrollo de la lexicografía pedagógica que se expondrán en este trabajo a partir de algunos ejemplos.

Palabras clave: Lexicografía pedagógica, lexicografía bilingüe, lexicografía online, diccionario onomasiológico, $\mathrm{DaF}$, Ele

Abstract: The analysis of existing bilingual lexicographical works for German and Spanish confirmed us the necessity to develop a dictionary with a novel approach, 
with which the user could receive a more tailored information to his needs, in case to produce texts in the respective foreign languages and which allows him to select a particular lexeme from the variety of possible lexicalizations, taking into account the diversity in their use. Based on this assumption, the contribution presents, on the one hand, the theoretical and methodological aspects, and on the other hand, some exemplary results of the lexicographical DICONALE online project. This is an ongoing project for the development of a modular bilingual and bidirectional dictionary with a conceptual onomasiological focus and online access. This dictionary is intended to be a tool in the production process of the foreign languages German and Spanish (DaF / ELE), in the translation of both by a range of possible lexicalized forms of certain mental concepts and in the achievement of relevant lexicological information to its correct use. The contribution will focus, for example, on some issues concerning the structure of DICONALE on a medium and micro level, and on several description modules. In the following, the four main pillars concerning the type of users, the onomasiological conception, the empirical data, the description model and the contrastive point of view will be presented and exemplified.

Keywords: Online Lexicography, Onomasiological Dictionary, Bilingual Lexicography, Pedagogical Lexicography, DaF, Ele 


\section{Introducción}

Algunas encuestas recientes sobre el uso de diccionarios en entornos de alemán y/o español como lenguas extranjeras ( $\mathrm{DaF}$ y/o Ele) han podido demostrar el creciente uso de todo tipo de obras de consulta en línea dentro y fuera del aula (Domínguez Vázquez et alii 2013, Fernández Méndez et alii 2016, Meliss 2015d). Las ventajas que se vinculan a este tipo de acceso son diversas ${ }^{1}$.

El presente artículo muestra los aspectos más relevantes del proyecto lexicográfico DICONALE-online que surge como respuesta a la necesidad de ofrecer otras posibilidades actuales frente a los nuevos hábitos de uso en los entornos de DaF y/o Ele.

El proyecto de investigación DICONALE-online ${ }^{2}$ consiste en la elaboración de un diccionario en línea de lexemas verbales y deverbales, de tipo bilingüe-bidireccional de las lenguas alemana y española con un acceso conceptual-onomasiológico. Esta obra de consulta está dirigida a usuarios que aprenden alemán o español como lengua extranjera, con un nivel avanzado (B2) y está concebida como un diccionario pedagógico ${ }^{3}$, de apoyo al usuario especialmente en los procesos de producción de textos en lengua extranjera (libre producción y traducción) ${ }^{4}$. La justificación del proyecto se encuentra en la existencia de numerosos estudios en los que se ha podido demostrar que los diccionarios convencionales pedagógicos para Ele y $\mathrm{DaF}$ (monolingües y bilingües), en formato print y/u online, no satisfacen las necesidades específicas de los usuarios en el ámbito referente a la producción de textos en lengua extranjera (cf. Fernández Méndez 2014, Fuentes Morán 1997, 84; Meliss 2013a, 2013b, 2014a, 2015b). De ese modo, el proyecto surge de la necesidad de cubrir una laguna existente en la lexicografía pedagógica bilingüe alemán-español y propone un diccionario con una orientación conceptual-onomasiológica que ofrezca una ayuda más adecuada en el proceso de búsqueda y selección de las posibles formas de expresión según el contexto

1 Para más información sobre estudios empíricos sobre el uso de los diccionarios véase la obra de MüllerSpitzer (ed.) (2014).

2 Este estudio se integra en el marco del proyecto de investigación DICONALE-online: «Elaboración de un diccionario conceptual bilingüe del alemán y del español: recurso online» (MINECO-FEDER FFI-201232658), dirigido por la Prof ${ }^{a} \operatorname{Dr}^{a}$ M. Meliss en la Universidad de Santiago de Compostela (<https://www. usc.es/es/proxectos/diconale/>) y está vinculado con la Rede de Lexicografía (Relex: <http://relex.udc.es/>: Xunta de Galicia: R/2014/042).

3 Cf. Porto Dapena 2002, 74.

4 Existen, entre otros, los siguientes diccionarios pedagógicos: para el alemán: el diccionario de Kempcke (KdG-DaF 2000), el de Pons DaF (P-DaF 2004: print+digital+online), el diccionario de Duden (D-DaF ${ }^{2} 2010$ ), el de Wahrig (W-DaF 2008), el de Götz et alii (LGWB-DaF ${ }^{3} 2010$ ); para el español: el diccionario de español para extranjeros de la editorial SM (Maldonado (dir.), 2002 = DSM-Ele), el Diccionario de Alcalá (Alvar Ezquerra (dir.), 1995 = DA-Ele), el Diccionario Salamanca (Gutiérrez Cuadrado (dir.), 2007 = DSEle) y el diccionario electrónico en construcción DAELE; 
y, posteriormente, para su uso correcto. Difiere así de la concepción tradicional alfabético-semasiológica que plantea la mayoría de los diccionarios pedagógicos.

El proyecto se basa en un modelo de descripción modular-integrativo y bilingüe-bidireccional 5 . El especial interés en la presentación sistemática de las estructuras tanto paradigmáticas como sintagmáticas de diferente índole se justifica sobre todo por las divergencias inter-e intralinguales entre lexemas con un significado parecido y por la necesidad de disponer de información detallada para la selección de un lexema entre la posible variedad y su uso adecuado posterior (González Ribao / Meliss 2015; González Ribao / Proost 2015). En este caso, hay que incidir en la carencia de diccionarios, sobre todo del español como lengua extranjera, que ofrezcan información sintagmática suficiente para situaciones de producción ${ }^{6}$.

Figura 1: Los cuatro pilares de DICONALEः (i) situación de uso, (ii) acceso, (iii), fundamentos teóricos y metodológicos y (iv) lenguas de partida y llegada en contraste.

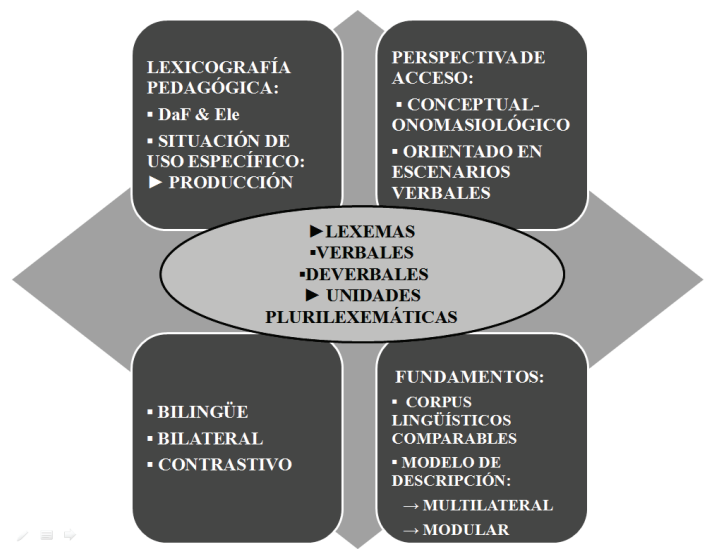

En este estudio se presentará la información más relevante sobre los cuatro pilares de DICONALE (cf. figura 1) y se incidirá en diversos aspectos como son la situación de uso y el tipo de usuario ( $\mathrm{DaF}$ y Ele, apartado 1), la perspectiva de acceso y el enfoque conceptual-onomasiológico (apartado 2), los fundamentos

\footnotetext{
5 En una posterior fase del proyecto se contemplará un enfoque multilingüe.

6 Desde los años 70 se observa un mayor interés por los diccionarios sintagmáticos. Entre estos, destacan los diccionarios que proponen información sobre aspectos sintácticos. De especial mención son para el español los diccionarios de Cuervo (1953/1998), Seco et alii ( ${ }^{1999), ~ B o s q u e ~(2004) ~ y ~ D i C E . ~ P a r a ~ e l ~ a l e m a ́ n ~ s o n ~ d e ~}$ especial interés los diccionarios de valencias que se han desarrollado en torno a las escuelas de Mannheim (Engel/Schumacher 1978, Schumacher et alii. 1986, Schumacher et alii Valbu 2004) y Leipzig/Berlín (Helbig/ Schenkel 1969, Sommerfeldt/Schreiber $\left.1974 /{ }^{3} 1983,1977 /{ }^{3} 1983,1996\right)$ y los diccionarios de colocaciones (Duden $2{ }^{9}$ 2010, Quasthoff 2011).
} 
teóricos y metodológicos, tales como la base empírica de los datos (apartado 3.1) y el modelo de descripción (apartado 3.2) y el punto de vista contrastivo bilateral (apartado 4). Al no existir, hasta ahora, obras lexicográficas del tipo propuesto por nosotros, el desarrollo del proyecto DICONALE supone un desafío para la disciplina en el contexto alemán-español ${ }^{7}$ y nos sitúa ante nuevos retos que se asocian a los cuatro pilares de dicho proyecto y enlazan parcialmente con las reflexiones de Tarp (2012).

\section{Usuarios}

Uno de nuestros principales retos es la creación de un diccionario más adaptado a las necesidades concretas del usuario. DICONALE es un diccionario pedagógico bilingüe que atiende especialmente a las necesidades de producción en lengua extranjera (L2) a partir del nivel B2. Las cuestiones del usuario que motivarán la consulta serán pues las siguientes:

- Búsqueda: ¿Qué recurso léxico existe en la L2 para la idea/el concepto que quiero expresar?

- Selección: ¿Cuál de los posibles recursos léxicos de la lengua de llegada se adapta mejor a la situación comunicativa?

- Uso: ¿Qué información es especialmente relevante desde un punto de vista contrastivo? Divergencias entre la lengua de partida y de llegada.

Las respuestas a estas cuestiones se centran en prestar una mayor atención a la información que se le suministra al usuario en su búsqueda de recursos léxicos, posteriormente, a la selección de uno u otro lexema y a la detallada descripción del potencial combinatorio - entre otros parámetros - para un uso apropiado. En diferentes estudios sobre diccionarios pedagógicos, tanto monolingües del alemán y del español, como bilingües de ambas lenguas, se ha podido demostrar que la información que la obra lexicográfica le ofrece al usuario en situaciones de producción es escasa e insuficiente. Así, a pesar de los enormes esfuerzos para introducir información explícita sobre los esquemas oracionales en los diccionarios monolingües de $\mathrm{DaF}$, en general, siguen existiendo dos necesidades fundamentales: (i) hacer más hincapié en los parámetros que definen el potencial combinatorio de un lexema y (ii) ofrecer más información sobre los parámetros

7 Hasta ahora, existen con esta perspectiva, para el español los diccionarios visuales de Alvar Ezquerra (Duden/Oxford 1993) y multilingüe de Pons (2008). Asimismo, son de mencionar el enfoque conceptual del diccionario de colocaciones DiCe (cf. Alonso Ramos 2004) y de ADESSE (cf. García Miguel et alii 2010). Además está prevista una parte onomasiológica en el Diccionario de la Coruña (cf. Porto Dapena et alii 2008). Para el alemán se pueden nombrar, entre otros, los siguientes: Duden Bildwörterbuch (2005), el diccionario para alemán como lengua extranjera de Kempcke 2000 (KdG-DaF) y las obras lexicográficas para DaF de Schreiber et alii. ${ }^{2} 1990,1991,1993$. 
distintivos entre lexemas semánticamente próximos, a fin de facilitar la selección de uno u otro, según el contexto (Meliss 2015b). Los diccionarios monolingües del español con fines pedagógicos también carecen en gran medida de información suficiente y satisfactoria para situaciones de producción (Meliss 2014a) ${ }^{8}$. Por otra parte, los diccionarios bilingües del alemán y del español ${ }^{9}$ han demostrado igualmente carencias en la información sintagmática (Fuentes Morán 1997, Meliss 2013a, Model 2010) a la vez que se ha puesto en duda su auténtico carácter bilingüe-bidireccional (Engelberg/Lemnitzer ${ }^{4} 2009,129$ ss. y 210ss. ${ }^{10}$. Ante la falta de este tipo de información, «nuestro usuario» recurre cada vez más a la oferta lexicográfica online que le permite - mediante un complejo sistema de hipervinculación - navegar, tanto por otros diccionarios mono- y bilingües del alemán y español como por portales lexicográficos que incluyen - entre otrosalgunos diccionarios específicos (diccionarios paradigmáticos y sintagmáticos, de construcción etc.). A pesar de las múltiples ventajas que ofrecen los diversos recursos online, no siempre son adecuados para el perfil del usuario que aquí nos interesa, ya que — en general - no están concebidos para usuarios de L2 y, por ello, su información resulta en general demasiado compleja para contextos de DaF y/o Ele (Meliss 2013b, 2014a, 2015b, 2015e). Para que el usuario del diccionario encuentre para sus fines la solución adecuada, DICONALE pretende ofrecer, de forma explícita, información contrastiva que pueda ser relevante, con el fin de seleccionar de la variedad existente, una u otra posibilidad de lexicalización en la lengua de llegada.

\section{El enfoque conceptual-onomasiológico}

\subsection{Antecedentes}

A diferencia de los diccionarios semasiológicos, los diccionarios onomasiológicos están esencialmente dirigidos a la producción (Reichmann 1989, Martín Mingorance 1994). Los diccionarios clásicos onomasiológicos para el alemán (Wehrle / Eggers 1961, Dornseiff 1965, Dornseiff / Quasthoff ${ }^{8} 2004$ ), así como p.ej. el diccionario ideológico de Casares (2007) para el español, si bien son las obras cumbre de la lexicografía onomasiológica, adolecen de falta de transparencia en los criterios de estructuración y de una información suficientemente detallada.

8 Como excepción se puede mencionar el diccionario de aprendizaje para Ele, DAELE, elaborado por la profesora Battaner Arias y su equipo (2008); véanse también Bernal / Renau (2010), Renau / Battaner (2012).

9 Para aspectos generales acerca de la lexicografía bilingüe del alemán y del español véanse Fuentes Morán (1997), Werner (1998), Haensch / Omeñaca (2004) y Hausmann (1991).

10 Para más información sobre el perfil del usuario y la descripción de las situaciones de uso véanse Egido Vicente et alii (2015) y Fernández Méndez et alii (2015). 
Aunque ofrecen varias posibilidades de expresión cuando se trata de buscar un signifiant para un signifié concreto, no brindan al usuario los criterios suficientes para la selección de un término y obligan, además, al uso de otros diccionarios para verificar el significado y su combinatoria (Meliss 2005, 65ss.). Dichas deficiencias justifican la necesidad de buscar nuevas vías lexicográficas para la perspectiva conceptual-onomasiológica. En este contexto son de mencionar, entre otras, las propuestas de estructuración de partes del léxico que enlazan con los presupuestos teóricos de la semántica estructural y la teoría del campo léxicosemántico (Coseriu 1977/v.esp. ${ }^{2}$ 1986; Geckeler 1971/ ${ }^{3} 1982$; Trujillo 1970). El punto de partida de estos estudios es la estructuración paradigmática de una serie de lexemas, relacionados entre sí mediante rasgos semánticos comunes, que lexicalizan conceptos afines y que, en parte, combinan la estructuración paradigmática con información sintagmática ${ }^{11}$. En consecuencia, desde los años ochenta, han aparecido algunos diccionarios y otras obras de consulta léxico-gramatical con una perspectiva conceptual-onomasiológica que presentan una descripción sistemática del léxico y combinan la estructuración paradigmática con una información sintagmática basada - en parte - en la teoría de valencias (Schumacher et al. 1986, Harras et al. 2004\&2007) y con una orientación especialmente enfocada hacia el uso en el entorno DaF (Schreiber et alii 1987/21990, 1991, 1993). También existen recientes estudios lexicográficos del español, que ofrecen información desde ambas perspectivas - la paradigmática y la sintagmática- (p.ej. ADESSE, DiCE y el diccionario de La Coruña) (cf. Meliss / Sánchez Hernández 2016).

\subsection{La propuesta de DICONALE}

El usuario accede al diccionario DICONALE mediante un doble marco de referencias conceptuales que constituye el tertium comparationis entre ambas lenguas. El primero consiste en una serie de unidades conceptuales - en parte, sustentado por elementos visuales-, que muestra una especificación conceptual cada vez mayor, lo que le permite al usuario elegir entre los campos conceptuales y, a su vez, entre subcampos de diferentes grados, según su intención comunicativa. A su vez, se ofrecen determinados escenarios verbales asociados a los (sub)campos conceptuales (Boas / Dux 2013, Subirats 2009) que configuran el segundo marco de referencia. Los lexemas asociados a unos conceptos determinados y

11 En cuanto a estudios que parten desde una perspectiva onomasiológica y un principio de estructuración léxica del alemán y español en contraste mediante principios paradigmáticos en combinación con información sintagmática, son de mencionar, entre otros, los estudios recientes de González Ribao / Proost (2015), Meliss (2005, 2015c), Sánchez Hernández (2010, 2012, 2015), Egido Vicente (2015), Fernández Méndez (2015), González Ribao (2015b). 
a un mismo escenario verbal se agrupan en diferentes (sub)paradigmas léxicosemánticos y se diferencian unos de otros por la existencia de rasgos semánticos distintivos, diferentes estructuras argumentales y diferentes realizaciones morfosintácticas y semánticas de los argumentos en cuestión. De esa manera, se pueden observar las divergencias y convergencias entre unos y otros agrupados en un mismo (sub)paradigma léxico-semántico.

En este sentido, el usuario realiza su búsqueda a partir de los conceptos / unidades conceptuales y no a partir de los lemas y de sus acepciones, y solo podrá acceder a las diferentes acepciones una vez que haya seleccionado las posibles lexicalizaciones para el concepto / las unidades conceptuales que haya buscado. Este recorrido de búsqueda se complementa con la selección de diferentes escenarios verbales y guía al usuario desde un concepto y un escenario verbal hacia diferentes posibilidades de lexicalización para las que se le ofrece información que facilita la selección y el uso posterior (cf. figura 2).

En una primera fase de trabajo, nos interesan los lexemas verbales simples y afijados y también —aunque de modo secundario - las formas plurilexemáticas del alemán y del español (Meliss 2014b, 2015a, Sánchez Hernández 2013a) ${ }^{12}$.

En consonancia con lo que apunta Haß-Zumkehr $(2001,264)$, la estructuración conceptual-onomasiológica del léxico será completada con la estructuración de los elementos atendiendo a sus relaciones paradigmáticas de significado. Ello supondrá un parámetro diferencial del modelo de descripción de DICONALE.

Figura 2: El recorrido de una búsqueda conceptual-onomasiológica en DICONALE

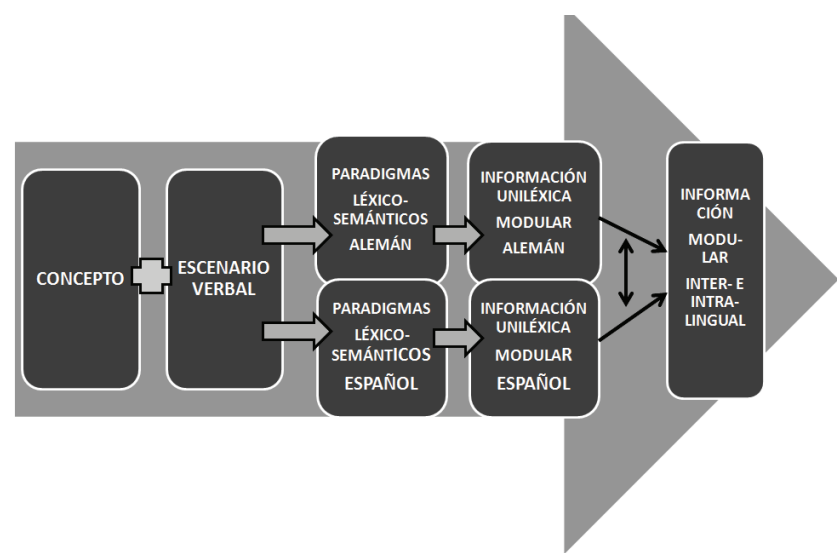

12 En una siguiente fase de trabajo está previsto ampliar el número de campos conceptuales y enriquecer la perspectiva bilingual con una perspectiva multilingual. 
La elaboración de un diccionario conceptual-onomasiológico, en los términos propuestos para DICONALE, supondrá una gran novedad en el marco de la lexicografía de aprendizaje del alemán y del español como lenguas extranjeras (cf. Meliss / Sánchez Hernández 2016). El acceso conceptual-onomasiológico se completará con un acceso semasiológico ante una consulta ordenada alfabéticamente. En tal caso, el usuario siempre será reconducido posteriormente mediante la oferta de las unidades conceptuales hacia una búsqueda conceptual-onomasiológica, tal y como indica la figura 3. Nuestro reto es la creación de los mecanismos que faciliten el acceso a la información desde una perspectiva conceptualonomasiológica - perspectiva bastante inusual — en el ámbito de la lexicografía pedagógica.

Figura 3: La combinación de ambos recorridos de búsqueda en DICONALE: acceso por orden alfabético y por búsqueda conceptual-onomasiológica.

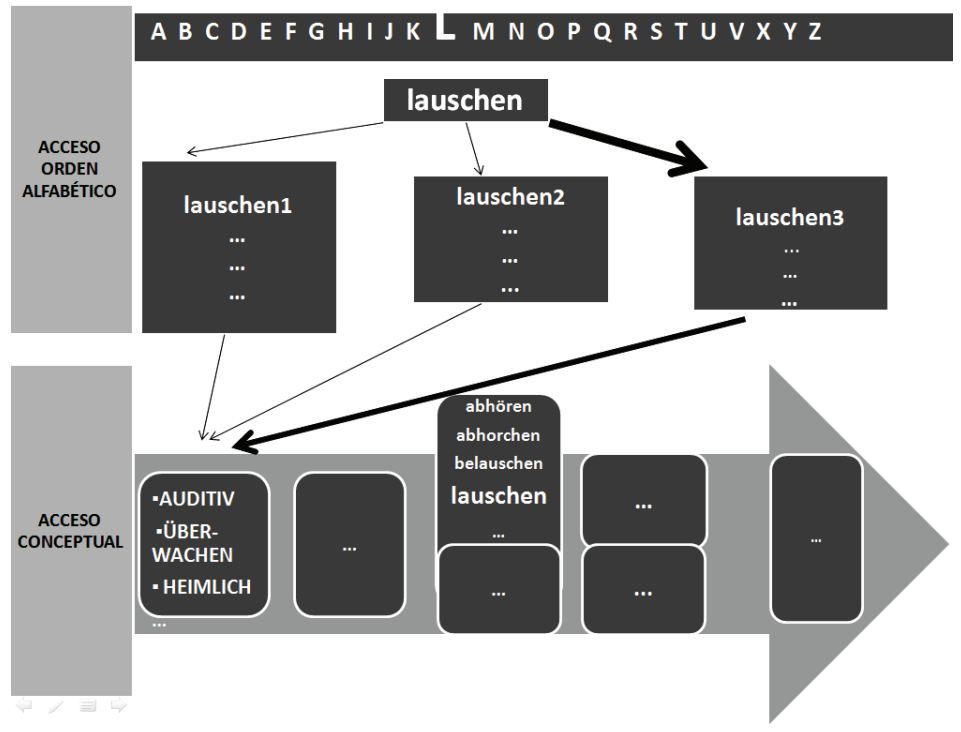

\section{Fundamentos metodológicos y teóricos}

\subsection{Base empírica}

La necesidad de basarse en corpus lingüísticos y cálculos de las frecuencias de uso para seleccionar la información ${ }^{13}$ queda patente en el estudio piloto, basado en el corpus de referencia del alemán DEREKO, sobre la información sintagmática re-

13 Cf. Bubenhofer et alii (eds.) (2010), Lemnitzer / Zinsmeister (2006), Renouf (ed.) (2009), Schmidt (ed.) (2012). 
lativa a las diferentes acepciones del verbo alemán abbören ${ }^{14}$ (Meliss 2015b). Los resultados del análisis del corpus, en comparación con la información de cinco diccionarios pedagógicos de alemán como lengua extranjera ${ }^{15}$, revelan que existe no solamente una diferencia informativa considerable entre los cinco diccionarios consultados, sino también una discrepancia respecto a los datos extraídos mediante el análisis. Para poder seguir un criterio uniforme, con el fin de seleccionar la información que se pretende ofrecer en DICONALE, es necesario trabajar de forma rigurosa con un método empírico válido. Para ello, hemos construido nuestro propio corpus de trabajo basado en textos de prensa de los corpus de referencia del alemán DEREKO y del español CREA y CORPES XXI (cf. González Ribao 2015a). De este modo se garantizan dos corpus de trabajo comparables para ambas lenguas que avalan el máximo grado de comparabilidad.

El proceso de codificación de la información de los corpus se reducirá a un número representativo de ejemplos para cada lengua que posteriormente será analizado manualmente según los parámetros predefinidos en el modelo de descripción. Entendemos que la evidencia empírica no solo forma la base para la selección de la información que pretende aportar DICONALE, sino también la justificación para la descripción de fenómenos menos frecuentes que - en muchos casos- quedan excluidas de las obras de consulta pedagógicas.

\subsection{Los fundamentos teóricos}

El modelo de descripción mediante el cual pretendemos codificar, analizar e interpretar la información para DICONALE desde los puntos de vista inter-e intralingual comprende cuatro módulos de descripción lexicológica y se organiza en cinco niveles ( $\mathrm{cf}$. Anexo). Mediante los diferentes parámetros de descripción (nivel 2) se codifica detalladamente la información lexicológica de forma modular (cuatro módulos) ( $\rightarrow$ microestructura). Esta información será la base tanto para los diferentes tipos de análisis a nivel 4 y 5 ( $\rightarrow$ medioestructura) como para los niveles 1 y 3 que, mediante complejas interrelaciones, aportan los datos para la estructuración conceptual y la organización del material léxico en (sub)campos léxico-semánticos ( $\rightarrow$ macroestructura). Tomando como base la información codificada e interrelacionada de los cinco niveles, nuestro reto es el desarrollo de una interfaz adecuada que permita un sistema flexible de acceso a la información del diccionario y adaptable a las diferentes necesidades del usuario (cf. figuras 2 y 3$)^{16}$.

14 Algunas correspondencias en español serían, entre otras: auscultar, escuchar, examinar, controlar, interceptar, intervenir, etc.

15 Para el citado análisis se han utilizado los cinco diccionarios pedagógicos de alemán como lengua extranjera, ya citados en la nota 6.

16 Cf. en alemán: Sánchez Hernández 2013b, Meliss 2014b, González Ribao / Meliss 2015; en español: Meliss 2015a, Fernández Méndez et alii 2015; en inglés: Egido Vicente et alii 2015. 
A partir de algunos ejemplos ilustrativos de los campos conceptuales AUDICión y COGNICIÓN se presentan, a continuación, algunos aspectos relevantes para la codificación de la información léxica. La interrelación de la información a nivel 1 permite agrupar los lexemas correspondientes en los diez campos (CC) y sus diferentes (sub)campos conceptuales (SCC) que actualmente se están elaborando. Las interrelaciones conceptuales y léxico-semánticas establecidas en este nivel, junto con las del nivel 3 (en el que se procede, por una parte, a una subclasificación conceptual de otros grados mediante una especificación según los escenarios verbales involucrados, y por otra, a la elaboración de los diferentes (sub)paradigmas léxico-semánticos (SPls)), formarán la MACroestructura conceptual del diccionario. La figura 4 ejemplifica dicha estructura de subcampos conceptuales de diferentes grados relacionados a la COGNICIón y vincula diferentes lexemas de ambas lenguas a los posibles paradigmas léxico-semánticos.

Figura 4: Subcampos conceptuales $2^{\circ}$ grado $\left(\mathrm{SCC}_{\mathrm{G} 2}\right)$ y lexicalización: subparadigmas léxico-semánticos $(\mathrm{SP})$ correspondientes a la lista de lexemas correspondientes ejemplificado mediante el subcampo conceptual $\left(\mathrm{SCC}_{\mathrm{G1}}\right)$ CogNición (secuencia).

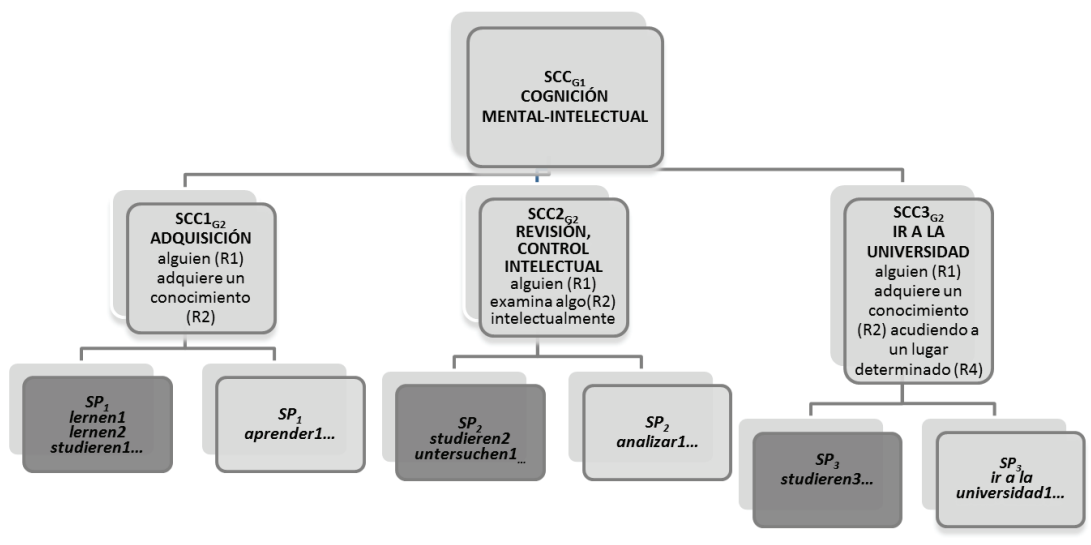

En el nivel 2 del modelo DICONALE se codifican para cada lengua por separado, mediante cuatro módulos, los datos lexicológicos detallados de cada lema y sus diferentes acepciones asociados a cada uno de los 10 campos conceptuales (nivel 1) y subcampos (nivel 3). El módulo 1 comprende, entre otros, datos generales que describen el aspecto formal (pronunciación, tipo de conjugación, rasgos suprasegmentales etc.) y de contenido (rasgos semánticos del lema etc.), mientras que el módulo 2 contempla las diferentes acepciones de cada lema a través de una 
descripción semántica que presenta, entre otros, los rasgos semánticos distintivos y las diferentes relaciones paradigmáticas de sentido, conjuntamente con la descripción de los diferentes roles correspondientes a los diferentes escenarios verbales, así como la información pragmática. En el módulo 3 se especifica la información sintagmática mediante los datos basados en la frecuencia empírica acerca de cada argumento y sus características morfosintácticas y semánticas ${ }^{17}$ (cf. Engelberg et alii 2012, Engelberg 2015a, 2015b), a la vez que se ofrece un posible equivalente en la lengua de contacto para cada acepción. La detallada información semántica, funcional y morfosintáctica relacionada con cada argumento ${ }^{18}$ proporciona los matices específicos en los que se basan muchas de las divergencias léxicas a nivel inter-e intralingual y a los cuales se les presta especial atención ${ }^{19}$. En el nivel 4 relacionamos, según diferentes enfoques, los resultados de los análisis respecto a los diferentes parámetros de descripción de los módulos centrales 2 y 3 del nivel 2. Esta información se presta a la posible presentación de diferentes tipos de (sub)paradigmas léxicos que configurarán la medioestructura del diccionario. Diferenciamos las siguientes interrelaciones entre los datos interlinguales: El nivel 4.1.1 (para el alemán) y 4.2.1 (para el español) interrelaciona y contrasta las diferentes acepciones de un lema asociado a un mismo (sub)campo conceptual, no solamente mediante información semántica en base a los rasgos semánticos distintivos (nivel 2: módulo 2) y la información sobre las posibles relaciones paradigmáticas de sentido, sino también respecto a las estructuras argumentales específicas, a las que se asocian informaciones morfosintácticas (nivel 2: módulo 3) y pragmáticas. Normalmente, las diferencias en la estructura semántica y en las estructuras argumentales $(=\mathrm{EARG})$ y oracionales $(=\mathrm{EO})$ permiten atribuir las diferentes acepciones a diferentes (sub)paradigmas léxicosemánticos, como es el caso de estudiar1 y estudiar2 (cf. figura 5). Otros datos de interés como la frecuencia de uso, los ejemplos ilustrativos y la información sobre lexemas semánticamente próximos se pondrán también a disposición del usuario de forma estructurada y en consonancia con su interés.

17 Como son p. ej. las estructuras oracionales, valencia sintáctica y semántica, descriptores, colocaciones etc. 18 Véanse algunos estudios contrastivos basados en estructuras argumentales entre el alemán y el rumano (Cosma et alii (eds. 2014)).

19 Véanse algunos estudios piloto que contemplan una perspectiva conceptual en combinación con estructuras argumentales en contraste entre el alemán y español: Egido Vicente (2015), Fernández Méndez (2015), González Ribao (2015b), González Ribao / Proost (2015), Meliss (2015c) y Sánchez Hernández (2015). 
Figura 5: Codificación de la información: Acepciones de estudiar e información relativa a los módulos 2 y 3 del nivel 2 del modelo de descripción (secuencia).

\begin{tabular}{|c|c|c|c|c|c|c|c|c|c|c|c|c|}
\hline \multicolumn{13}{|c|}{ Campo conceptual COGNICIÓN } \\
\hline \multicolumn{13}{|c|}{ Subcampo conceptual LERNEN/APRENDER } \\
\hline \multicolumn{4}{|l|}{ ESTUDIAR } & \multicolumn{6}{|c|}{$\begin{array}{l}\text { Rasgos semánticos } \\
\text { distintivos }\end{array}$} & \multicolumn{3}{|c|}{ Argumentos } \\
\hline $\begin{array}{l}{[+ \text { cognición }]} \\
{[+ \text { adquisición }]} \\
{[+ \text { mental }]} \\
{[+ \text { intelectual }]}\end{array}$ & $\begin{array}{l}\text { (Sub) } \\
\text { para- } \\
\text { digma }\end{array}$ & Ejemplos & $\Sigma$ & $\begin{array}{l}S \\
1\end{array}$ & $\begin{array}{l}S \\
2\end{array}$ & $\begin{array}{l}S \\
3\end{array}$ & $\begin{array}{l}S \\
4\end{array}$ & $\begin{array}{l}S \\
5\end{array}$ & $\begin{array}{l}S \\
6\end{array}$ & $\begin{array}{c}\text { A } \\
1\end{array}$ & $\begin{array}{l}\text { A } \\
2\end{array}$ & $\begin{array}{c}\text { A } \\
3\end{array}$ \\
\hline $\begin{array}{l}\text { estudiar1 } \\
\text { estudiar }\end{array}$ & SP1 & & & + & & + & + & & + & & & \\
\hline $\begin{array}{r}\text { Alguien (A1) estudia algo (A2) } \\
\mathrm{EO}<\mathrm{s}(\mathrm{cd})>\end{array}$ & & & & & & & & & & s & (cd) & \\
\hline $\begin{array}{l}\text { estudiar2 } \\
\text { Alguien }(A 1) \text { estudia algo (A2) } \\
\text { en un lugar concreto }(A 3) \\
\qquad \mathrm{EO}<\mathrm{s}(\mathbf{c d})\left(\mathbf{c} \mathrm{p}_{\mathrm{en}}\right)>\end{array}$ & & & & & & & & & & s & (cd) & (cp) \\
\hline $\begin{array}{l}\text { estudiar3 } \\
\text { analizar intelectualmente } \\
{[+/ \text {-Erwerb] }}\end{array}$ & SP2 & & & & + & + & & + & + & & & \\
\hline $\begin{array}{l}\text { 区Alguien (A1) estudia algo } \\
\text { (A2) } \\
\qquad \text { EO }<\text { s cd }>\end{array}$ & & & & & & & & & & s & (cd) & \\
\hline Rasgos semánticos distintivos: & \multicolumn{12}{|c|}{$\begin{array}{l}\text { S1: [+disciplinal], S2: [+análisis], S3: [+concentración], S4: [+memoria], } \\
\text { S5: [+control], S6: [+proceso intelectual]. }\end{array}$} \\
\hline Complementos: & \multicolumn{12}{|c|}{$\begin{array}{l}\mathrm{s}=\text { sujeto, } \mathrm{cd}=\text { complemento directo, } \mathrm{cp}=\text { complemento prepositivo, }(\ldots) \\
=\text { facultativo }\end{array}$} \\
\hline $\begin{array}{l}\text { Argumentos: } \\
\text { Con descripción semántica- } \\
\text { categorial }[\ldots] \text { y descriptores } \\
\text { para su mayor especificación: }\end{array}$ & \multicolumn{12}{|c|}{$\begin{array}{l}\text { A1: ESTUDIANTE [+anim]; A2: LO ESTUDIADO: [+disciplina] } \\
\text { vocabulario, matemática; informe A3: LUGAR DE ESTUDIO: en la } \\
\text { escuela, biblioteca, etc. }\end{array}$} \\
\hline
\end{tabular}

El nivel 4.1.2 (para el alemán) y 4.2.2 (para el español) interrelaciona los lexemas de un mismo (sub)campo conceptual y permite la «creación» de diferentes paradigmas léxico-semánticos para ambas lenguas en base a los parámetros conceptuales y semánticos mencionados. A partir de esta estructuración se pueden analizar y codificar diversas relaciones paradigmáticas de significado entre los elementos de un mismo (sub)campo léxico-semántico o fuera de él. El usuario podrá realizar su selección atendiendo a diversos requisitos, como el tipo de texto, los recursos estilísticos, etc. para insertar el elemento lingüístico más adecuado en el texto (Sánchez Hernández 2013b).

Las relaciones paradigmáticas pueden observarse en torno a la definición semántica, o bien pueden formar parte de la entrada léxica de una manera independiente. La plasmación de la información paradigmática de una manera inde- 
pendiente en una entrada es lo que se conoce como intentionelle Paradigmatik, que supone una serie de prerrogativas en el diccionario (Hausmann 1991, 2794). Este tipo de información fomenta los procesos de producción y amplía el vocabulario, siempre y cuando se pueda relacionar con la información combinatoria correspondiente al nivel 2, módulo 3. La figura 6 presenta un ejemplo en torno al subcampo conceptual PERCEPCión AUdiCión y los diferentes subcampos de segundo grado, las posibles lexicalizaciones agrupadas en los paradigmas léxicosemánticos correspondientes y la indicación de lexemas que presentan algunas otras relaciones paradigmáticas de sentido.

Figura 6: Codificación de la información: Algunas relaciones paradigmáticas: Campo conceptual PERCEPCión-AUdiCión.

\begin{tabular}{|c|c|c|c|c|}
\hline $\begin{array}{c}\text { Campo } \\
\text { conceptual }\end{array}$ & $\begin{array}{c}\text { Subcampos conceptuales } \\
\text { del alemán }\end{array}$ & $\begin{array}{l}\text { Subparadigmas léxico- } \\
\text { semánticos del alemán }\end{array}$ & \multicolumn{2}{|c|}{$\begin{array}{c}\text { Relaciones paradigmáticas } \\
\text { en alemán }\end{array}$} \\
\hline \multirow[t]{5}{*}{$\begin{array}{l}\mathrm{SCC}_{\mathrm{G} 1} \\
\text { PERCEPCIÓN } \\
\text { AUDICION }\end{array}$} & $\begin{array}{l}\text { SCC5 }_{\mathrm{G} 2} \\
\text { HEIMLICH AUDITIV } \\
\text { ÜBERWACHEN // } \\
\text { VIGILAR EN SECRETO Y } \\
\text { DE FORMA AUDITIVA }\end{array}$ & $\begin{array}{l}\text { SP5 } \\
\text { abbören2 } \\
\text { abborchen3 } \\
\text { borchen2 } \\
\text { lauschen3 } \\
\ldots\end{array}$ & $\begin{array}{l}\text { Hiperonimia } \\
\text { kontrollieren } \\
\text { spionieren } \\
\text { überwachen } \\
\text {... }\end{array}$ & Antonimia \\
\hline & $\begin{array}{l}\mathrm{SCC}_{\mathrm{G} 2} \\
\mathrm{MIT}_{\mathrm{DEM}} \mathrm{GEHÖR} \\
\text { BEOBACHTEN / } \\
\text { ÜBERPRÜFEN / } \\
\text { KONTROLLIEREN // } \\
\text { OBSERVAR / VIGILAR / } \\
\text { COMPROBAR CON EL } \\
\text { OÍDO }\end{array}$ & $\begin{array}{l}\text { SP4 } \\
\text { bören } 3 \\
\text { borchen2 } \\
\text { lauschen2 } \\
\text { belauschen1 } \\
\text { abbören2 } \\
\text { abhorchen2 } \\
\ldots\end{array}$ & $\begin{array}{l}\text { Hiperonimia } \\
\text { beobachten } \\
\text { überprüfen } \\
\text { kontrollieren } \\
\ldots\end{array}$ & Antonimia \\
\hline & $\begin{array}{l}\mathrm{SCC}_{\mathrm{G} 2} \\
\text { BEWUSST AKUSTISCH } \\
\text { WAHRNEHMEN UND } \\
\text { MENTAL VERARBEIT // } \\
\text { PERCIBIR DE } \\
\text { FORMA CONCIENTE } \\
\text { YTRATARLO } \\
\text { MENTALMENTE }\end{array}$ & $\begin{array}{l}\text { SP3 } \\
\text { bören } 2 \\
\text { lauschen1 } \\
\text { zubören1 } \\
\text { (sich) anbören1 } \\
\text { horchen1 } \\
\ldots\end{array}$ & $\begin{array}{l}\text { Hiperonimia } \\
\text { wahrnehmen } \\
\text { aufnehmen } \\
\text { merken } \\
\text { verarbeiten } \\
\text { verstehen } \\
\text {... }\end{array}$ & $\begin{array}{l}\text { Antonimia } \\
\text { sehen } \\
\text { riechen } \\
\text { schmecken } \\
\end{array}$ \\
\hline & $\begin{array}{l}\mathrm{SCC}_{\mathrm{G} 2} \\
\text { MEDIZINISCH } \\
\text { UNTERSUCHEN // } \\
\text { REALIZAR UN ANÁLISIS } \\
\text { MÉDICO }\end{array}$ & $\begin{array}{l}\text { SP2 } \\
\text { abbören1 } \\
\text { abborchen1 } \\
\text { auskultieren \{med.\} } \\
\text {... }\end{array}$ & $\begin{array}{l}\text { Hiperonimia } \\
\text { untersuchen } \\
\ldots\end{array}$ & Antonimia \\
\hline & $\begin{array}{l}\text { SCC1 }_{\mathrm{G} 2} \\
\text { AKUSTISCH } \\
\text { WAHRNEHMEN // } \\
\text { PERCIBIR } \\
\text { ACUSTICAMENTE }\end{array}$ & $\begin{array}{l}\text { SP1 } \\
\text { bören1 } \\
\text { vernebmen1 } \\
\end{array}$ & $\begin{array}{l}\text { Hiperonimia } \\
\text { wabrnebmen } \\
\text { merken } \\
\text {... }\end{array}$ & $\begin{array}{l}\text { Antonimia } \\
\text { seben } \\
\text { riechen } \\
\text { schmecken } \\
\text {... }\end{array}$ \\
\hline
\end{tabular}

El nivel 4.1 .3 (para el alemán) y el 4.2 .3 (para el español) del modelo de descripción se dedica a codificar las interrelaciones de los diferentes lexemas de un 
mismo SP léxico-semántico (cf. nivel 3) en función de sus características semánticas y combinatorias (cf. nivel 2: módulos 2 y 3 ). En el centro del interés se encuentra la información detallada acerca de los diferentes argumentos, su posible materialización mediante actantes oracionales y sus realizaciones semánticas y morfosintácticas. Además se ofrecen posibles equivalencias en la lengua de contacto (cf. figura 7).

\section{Figura 7: Codificación de la información: Nivel 4.1.3ः Lexemas correspon-} dientes al SP 3 con la información parcial correspondiente al nivel 2 (módulos 2 y 3 ) (secuencia) (para el metalenguaje véase figura 5 ).

\begin{tabular}{|c|c|c|c|c|c|c|c|c|}
\hline \multicolumn{9}{|c|}{ Campo conceptual Cognición } \\
\hline \multirow{2}{*}{\multicolumn{9}{|c|}{ Subcampo conceptual Adquisición: intelectual }} \\
\hline & & & & & & & & \\
\hline \multicolumn{9}{|c|}{ Subparadigma léxico-semántico: cognición SP1 } \\
\hline & & & & & & & \multicolumn{2}{|c|}{$\begin{array}{r}{[+ \text { mental }]} \\
{[\text { +apropiación }]}\end{array}$} \\
\hline & \multirow{2}{*}{ 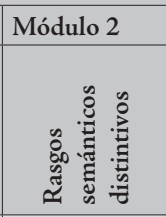 } & \multicolumn{7}{|c|}{ Módulo 3} \\
\hline & & 范 & $\begin{array}{l}8 \\
\text { w } \\
\text { W }\end{array}$ & ¿ & 2 & $\stackrel{m}{\gtrless}$ & 走 & 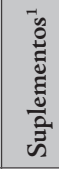 \\
\hline aprender1 & $\varnothing$ & & & & & & & \\
\hline $\begin{array}{l}\text { ASTM1 } \\
\text { alguien (A1) } \\
\text { aprende algo (A2) } \\
\qquad \begin{array}{l}\text { EO }<\text { s cd }> \\
\rightarrow \text { lernen } 1\end{array}\end{array}$ & & & & $\begin{array}{l}\text { s } \\
{[+ \text { hum }]} \\
{[+ \text { zool }]} \\
\ldots\end{array}$ & $\begin{array}{l}\mathrm{cd} \\
{[+ \text { discipl }]} \\
{[+ \text { acción }]} \\
{[+ \text { mat }]}\end{array}$ & & & $\cdots$ \\
\hline $\begin{array}{l}\text { ASTM2 } \\
\text { alguien (A1) } \\
\text { aprende algo (A2) } \\
\text { de algo(A3) o de } \\
\text { alguien (A4) } \\
\qquad \mathrm{EO}<\text { s cd cp }> \\
\quad \rightarrow \text { lernen2 }\end{array}$ & & & & $\begin{array}{l}\text { s } \\
{[+ \text { hum }]} \\
{[+ \text { zool }]} \\
\cdots\end{array}$ & $\begin{array}{l}\text { cd } \\
{[+ \text { cogn }]} \\
{[+ \text { situación }]}\end{array}$ & \begin{tabular}{|l} 
cp - de \\
{$[-$ hum $]$} \\
{$[+$ cogn $]$} \\
{$[-$ zool $]$} \\
Vida, \\
carrera
\end{tabular} & $\begin{array}{l}\text { cp }- \text { de } \\
\text { [+hum] } \\
\text { [+zool] } \\
\text { padres } \\
\text { profesor, } \\
\text { jefe, } \\
\text { animales... }\end{array}$ & \\
\hline $\begin{array}{l}\text { ASTM3 } \\
\text { Alguien (A1) } \\
\text { aprende algo (A2) } \\
\text { de algo (A3) } \\
\text { EO }<\text { s cd cp }> \\
\rightarrow \text { lernen3 }\end{array}$ & & & & $\begin{array}{l}\text { s } \\
{[+ \text { hum }]} \\
{[+ \text { zool }]}\end{array}$ & $\begin{array}{l}\text { cd } \\
{[+\operatorname{cogn}]} \\
{[+ \text { situación }]}\end{array}$ & $\begin{array}{l}\text { cp -de } \\
{[- \text { hum }]} \\
{[+ \text { cogn }]} \\
{[- \text { zool }]} \\
\text { errores, } \\
\text { experiencia } \\
\text { historia... } \\
\end{array}$ & & \\
\hline empollar & $\begin{array}{l}\text { [+intensiv }] \\
{[+ \text { coloquial }]}\end{array}$ & & & & & & & \\
\hline $\begin{array}{l}\text { ASTM2 } \\
\text { alguien (A1) } \\
\text { empolla algo (A2) } \\
\text { EO }<\text { s cd }> \\
\rightarrow \text { pauken } 1 \\
\end{array}$ & & & & $\begin{array}{l}\text { s } \\
{[+ \text { hum }]} \\
\ldots\end{array}$ & $\begin{array}{l}\text { cd } \\
\text { [discipl] } \\
\text { lección, } \\
\text { vocabulario } \\
\ldots\end{array}$ & & & $\ldots$ \\
\hline$[\ldots]$ & & & & & & & & \\
\hline
\end{tabular}




\section{Las lenguas en contraste}

Otro de nuestros retos es la creación de los mecanismos para darle más visibilidad a los contrastes inter-e intralinguales, ya que los diccionarios mono- y bilingües no inciden, en general, de forma explícita en ellos. El nivel 5 del modelo de descripción se ocupa de la información interlingual. Hay dos enfoques comparativos de interés. En primer lugar, se contrastan subparadigmas léxico-semánticos de ambas lenguas que corresponden al mismo (sub)campo-concepto. Para ello nos servimos sobre todo de las interrelaciones a nivel de cada SP léxico semántico (cf. nivel 3) y las contrastamos con la lengua en contacto. Se trata de comparar los diferentes datos lexicológicos a nivel de los campos léxico-semánticos, como el grado de lexicalización y otros (nivel 5.1). En segundo lugar, es de interés un estudio contrastivo entre los lexemas que lexicalizan los mismos conceptos y sus características lexicológicas determinadas (nivel 5.2). Es de esperar que las divergencias entre ambas lenguas se encuentren sobre todo en la configuración semántica (C1) y en las diferentes especificaciones morfosintácticas y semánticas de los diferentes argumentos $\left(\mathrm{C}_{1-\text {-xyzz }}\right)^{20}$. Pero también son de especial interés los datos de frecuencia y uso referentes a otros parámetros de los módulos 2 y 3 del nivel 2 del modelo DICONALE. La figura 8 visualiza a nivel 5.2 posibles contrastes a nivel semántico $(\mathrm{C} 1)$ y morfosintáctico $\left(\mathrm{C} 2{ }_{1}\right)$ entre lexemas del SP3 «zuhören/escuchar-Paradigma» vinculado al SCC AUDITION, partiendo del español. (i) Existen contrastes en la especificación semántica (C1): sich anbören1 y lauschen 1 se caracterizan por los rasgos distintivos [+exacto/genau] y [+concentrado/konzentriert]. (ii) Los lexemas zubören 1 y lauschen 1 poseen la misma estructura argumental (EARG) que escuchar2, pero la realización morfosintáctica del argumento A3 difiere (C2). Mientras en español se realiza mediante un cd, en alemán recurrimos a un dativo. La presentación desde diferentes perspectivas contrastivas permitirá al usuario encontrar los recursos léxicos adecuados a su necesidad de expresión en la lengua extranjera y el uso correcto de las mismas, ya que se incide especialmente y de forma estructurada y sistemática en las diferentes divergencias entre ambas lenguas.

20 C1 = contraste a nivel de la configuración semántica; C2 = contraste a nivel argumental; la especificación de los contrastes $\mathrm{C} 2$ se indica con superíndices. 


\section{Figura 8: Codificación de la informaciónः Nivel 5.2: Algunas posibles divergencias entre lexemas del SP3 vinculado al SCC PERCEPCióN: AUdición (secuencia).}

\begin{tabular}{|c|c|c|c|c|}
\hline \multicolumn{4}{|c|}{$\begin{array}{l}\text { Nivel de descripción 5.2.: contraste: divergencias y } \\
\text { convergencias }\end{array}$} & tertium comparationis \\
\hline \multicolumn{4}{|c|}{$\begin{array}{l}\text { PERCEPCIÓN ACÚSTICA Y PROCESAMIENTO } \\
\text { MENTAL CONSCIENTE } \\
\text { SP3: «zuhören/escuchar»-Paradigma }\end{array}$} & $\begin{array}{l}\text { [+percepción] } \\
{[+ \text { acústico }]} \\
{[+ \text { de forma consciente }]} \\
\text { [+procesar mentalmente }] \\
\text { +EARG|A1 A3| }\end{array}$ \\
\hline \multirow[t]{8}{*}{ escuchar2 } & & EO & $\begin{array}{l}\text { Rasgos } \\
\text { semánticos } \\
\text { distintivos }\end{array}$ & Ejemplos: \\
\hline & $\begin{array}{l}\text { Alguien (A1) } \\
\text { escucha algo (A3) }\end{array}$ & $\begin{array}{l}<s \\
(c d)>\end{array}$ & & $\begin{array}{l}\text { (1a) La prueba estuvo ayer en la sala pequeña } \\
\text { del teatro Dramaten, donde el público se rió } \\
\text { de lo lindo y escucbó atentamente la lectura de } \\
\text { dos pasajes de Infancia [...] (CREA) } \\
\text { (1b) Cuando escucho la música de mis colegas, } \\
\text { me gusta, lo paso bien oyéndola. (CREA) } \\
\text { (1c) Durante la visita a la fábrica, el } \\
\text { Rey escuchó las explicaciones de los hermanos } \\
\text { Puig sobre el proceso de producción de } \\
\text { colonias y perfumes [...] (CREA) }\end{array}$ \\
\hline & zubören 1 & & $\neq \mathrm{C} 2: \mathrm{A} 2$ & \\
\hline & $\begin{array}{l}\text { Jemand (A1) hört } \\
\text { etwas (A3) } z u\end{array}$ & $\langle\mathrm{~s} d>$ & $\neq \mathrm{C} 1$ & $\begin{array}{l}\text { (2) Mit großem Interesse hatten die } \\
\text { Pflegekräfte [...], dem Vortrag zugehört } \\
\text { [...]. M03/FEB.11701 Mannh. Morgen, } \\
\text { 22.02.2003 }\end{array}$ \\
\hline & (sich) anbören1 & & $\begin{array}{l}\text { [+con precisión / } \\
\text { genau] }\end{array}$ & \\
\hline & $\begin{array}{l}\text { Jemand (A1) } \\
\text { bört (sich) etwas } \\
\text { (A3) an }\end{array}$ & $<\mathrm{s}$ a $>$ & $\neq \mathrm{C} 1$ & $\begin{array}{l}\text { (3) Sie bören sich die Probleme an, die den } \\
\text { Kindern auf den Nägeln brennen [...]. (R97/ } \\
\text { SEP.72386 Frankf. Rundschau, 15.09.1997, } \\
\text { S. 4) }\end{array}$ \\
\hline & lauschen 1 & & $\begin{array}{l}{[+ \text { concentrado / }} \\
\text { konzentriert] }\end{array}$ & \\
\hline & $\begin{array}{l}\text { Jemand (A1) } \\
\text { lauscht etwas } \\
\text { (A3) }\end{array}$ & $\langle\mathrm{s} d>$ & $\neq \mathrm{C} 2: \mathrm{A} 2$ & $\begin{array}{l}\text { (4) In dem bis auf den letzten Platz gefüllten } \\
\text { Gotteshaus lauscbten mehr als } 2000 \\
\text { Besucher andächtig den Chorgesängen [...]. } \\
\text { (L98/NOV.16306 Berliner Morgenpost, } \\
01.11 .1998, \text { S. 9) }\end{array}$ \\
\hline
\end{tabular}

\section{Conclusiones}

Con la elaboración del diccionario DICONALE se intenta cubrir una laguna en la lexicografía pedagógica bilingüe alemán-español que se traduce en el desafío de querer integrar diversos tipos de información en el mismo diccionario y adaptarla a las necesidades de un usuario determinado y una situación específica, que es la de la producción y traducción en lengua extranjera. En el futuro, nuestra pro- 
puesta pretende convertirse en un sistema de consulta léxico-gramatical bilingüe con un especial enfoque contrastivo bilateral.

Este desafío nos lleva a asumir los retos a los que nos enfrentamos con la elaboración de este modelo y que hemos querido describir en esta contribución: $a$ ) una obra de consulta dirigida a la producción en un contexto de lexicografía pedagógica para $\mathrm{DaF}$ y Ele, $b$ ) desde una perspectiva conceptual u onomasiológica, c) con una ordenación bilingüe y bilateral, donde se contemple el punto de vista contrastivo y d) cuya compilación de entradas provenga de corpus lingüísticos reales. 


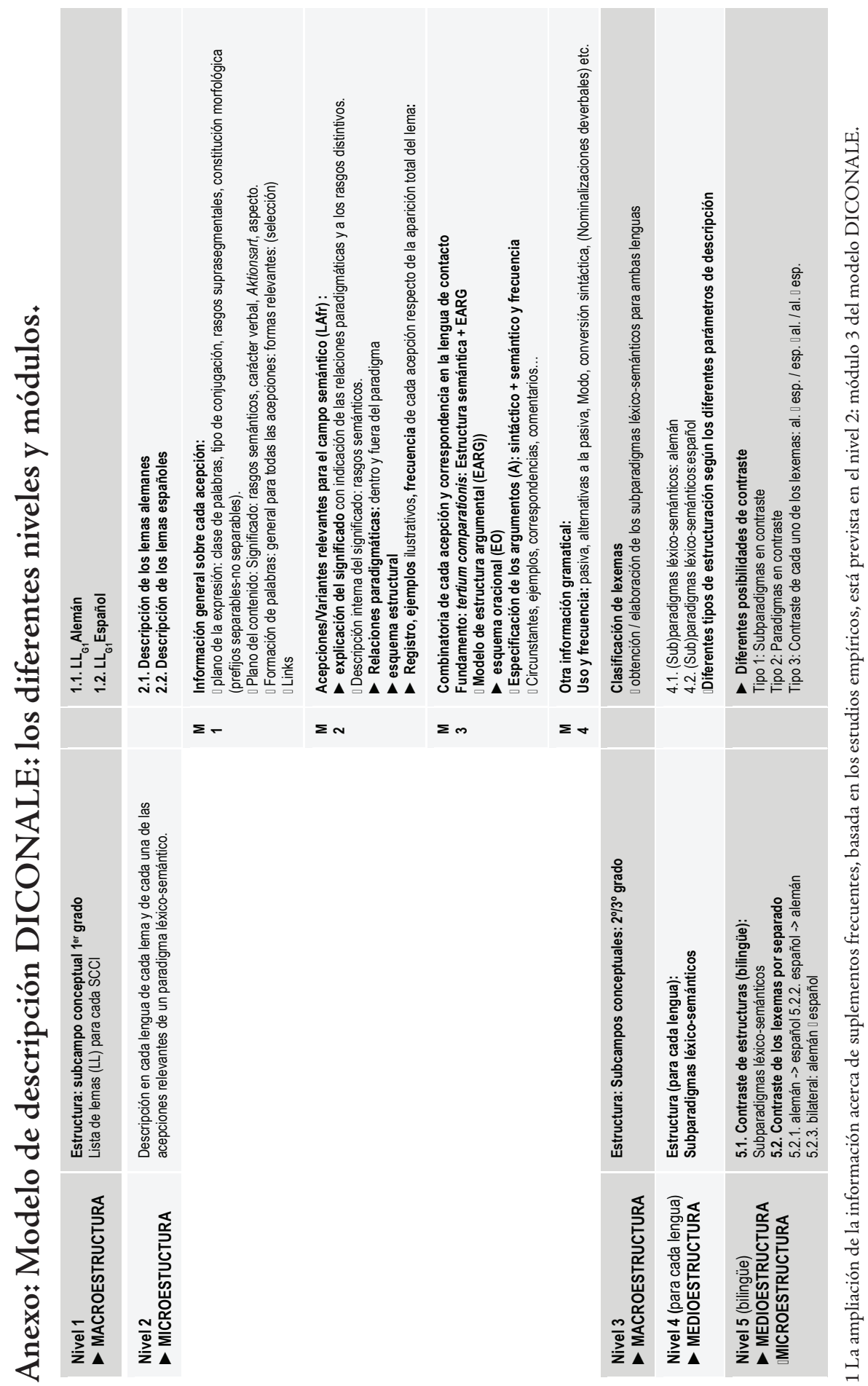




\section{Referencias bibliográficas}

Todos los recursos online se han consultado por última vez 15/02/2015.

\section{Diccionarios y corpus lingüísticos}

Adesse. Base de datos de Verbos, Alternancias de Diátesis y Esquemas SintácticoSemánticos del Español. [<http://adesse.uvigo.es/data/verbos.php >].

Alvar Ezquerra, M. (1993). Duden/Oxford Bildwörterbuch Deutsch und Spanisch. Mannheim: Duden.

Bosque, I. (2004). Redes: Diccionario combinatorio del español contemporáneo. Madrid: SM.

Casares, J. (2007). Diccionario ideológico de la lengua española. [1. Ed. 1942]. Barcelona: Gustavo Gili.

CORPES XXI. Corpus del español del siglo XXI. [<http://web.frl.es/CORPES/ view/inicioExterno.view $>$ ].

CREA. Real Academia Española: Banco de datos. Corpus de referencia del español actual. $[<$ http://www.rae.es $>]$.

Cuervo, R. J. (1953/1998). Diccionario de construcción y régimen de la lengua castellana. Barcelona: Herder.

DAELE. Diccionario de aprendizaje del español como lengua extranjera. [<http:// www.iula.upf.edu/rec/daele/>]

DA-Ele $\rightarrow$ Diccionario de Alcalá.

D-DaF: $\rightarrow$ Duden Deutsch als Fremdsprache.

DEREKO. Deutsche Referenzkorpus. [<http://www.ids-mannheim.de/kl/projekte/korpora/ > über COSMAS II: Corpus Search Management and Analysis System. [<http://www.ids-mannheim.de/cosmas2/>].

Diccionario de Alcalá: Alvar Ezquerra, M. (dir.). (1995/22000). Diccionario para la enseñanza de la lengua española. Español para extranjeros. Barcelona: Vox y Universidad de Alcalá. $\rightarrow$ DA-Ele.

Diccionario Salamanca: Gutiérrez Cuadrado, J. (dir.) (1996/2007). Diccionario Salamanca de la lengua española. Madrid: Santillana y Universidad de Salamanca $\rightarrow$ DS-Ele.

Diccionario SM: Maldonado, C. (dir.) (2002). Diccionario de español para extranjeros. Madrid: SM. $\rightarrow$ DSM-Ele.

DiCE: Diccionario de Colocaciones del Español. [<http://www.dicesp.com/paginas > ].

Dornseiff, F. (1965). Der deutsche Wortschatz nach Sachgruppen. [1. Auflage 1934]. Berlin: De Gruyter. 
Dornseiff, F. / Quasthoff, U. ( $\left.{ }^{8} 2004\right)$. Der deutsche Wortschatz nach Sachgruppen. Berlin: De Gruyter.

DS-Ele: $\rightarrow$ Diccionario de Salamanca.

DSM-Ele: $\rightarrow$ Diccionario SM.

Duden (2005). Bildwörterbuch Deutsch als Fremdsprache. Mannheim: Duden.

Duden 2 ( $\left.{ }^{9} 2010\right)$. Das Stilwörterbuch. Mannheim: Dudenverlag.

Duden Deutsch als Fremdsprache - Standardwörterbuch. (2010). Mannheim: Duden. $\rightarrow$ D-DaF.

Engel, U. / Schumacher, H. (1978.). Kleines Valenzlexikon deutscher Verben. Tübingen: G. Narr.

E-Valbu $\rightarrow$ Schumacher, H. et alii.

Götz, D. / Haensch, G. / Wellmann, H. ('2010). Langenscheidts Großwörterbuch Deutsch als Fremdsprache. Neubearbeitung: Berlin / München. Langenscheidt. $\rightarrow$ LGW-DaF

Harras, G. et alii (2004 \& 2007). Handwörterbuch deutscher Kommunikationsverben. Teil 1: Wörterbuch. Teil 2: Lexikalische Strukturen. Berlin: De Gruyter. Online: OWID - IDS: Kommunikationsverben: [<http://www.owid. $\mathrm{de} / \mathrm{docs} /$ komvb/start.jsp $>$ ].

Helbig, G. / Schenkel, W. (1969). Wörterbuch zur Valenz und Distribution deutscher Verben. Leipzigः VEB Bibliographisches Institut Leipzig.

Keмpске, G. (2000). Wörterbuch Deutsch als Fremdsprache. Berlin / New York: De Gruyter. $\rightarrow$ KdG-DaF.

LGW-DaF $\rightarrow$ GöTz, D. et alii.

Pons (2004). Großwörterbuch DaF. Stuttgart» Pons. Online: $[<$ http://de.pons. $\mathrm{eu} />$ ] $\rightarrow$ Pons-DaF.

Pons (2008). Das große Bildwörterbuch Deutsch, Englisch, Französisch, Spanisch, Italienisch. Stuttgart: Ernst Klett Sprachen.

Pons-DaF $\rightarrow$ Pons, Großwörterbuch DaF.

Quasthoff, U. (2011). Wörterbuch der Kollokationen im Deutschen. Berlin: De Gruyter + versión electrónica.

Schreiber, H. / Sommerfeldt, K. E. / Starke, G. (1987/2¹990). Deutsche Wortfelder für den Sprachunterricht. Verbgruppen. Leipzigः Langenscheidt Verlag Enzyklopädie.

Schreiber, H. / Sommerfeldt, K. E. / Starke, G. (1991). Deutsche Adjektive. Wortfelder für den Sprachunterricht. Leipzig: Langenscheidt Verlag Enzyklopädie.

Schreiber, H. / Sommerfeldt, K. E. / Starke, G. (1993). Deutsche Substantive. Wortfelder für den Sprachunterricht. Leipzig: Verlag Enzyklopädie. 
Schumacher, H. et alii (2004). Valbu-Valenzwörterbuch deutscher Verben. Tübingen. G. Narr. $\rightarrow$ E-Valbu [http://hypermedia.ids-mannheim.de/evalbu/ index.html].

Schumacher, H. et alii (1986). Verben in Feldern. Valenzwörterbuch zur Syntax und Semantik deutscher Verben. Berlin: De Gruyter.

Seco, M. / De Andrés, O. / Ramos, G. ( $\left.{ }^{3} 1999\right)$. Diccionario del español actual. Madrid: Aguilar.

Sommerfeldt, K. E. / Schreiber, H. (1974/ $\left.{ }^{3} 1983\right)$. Wörterbuch zur Valenz und Distribution deutscher Adjektive. Tübingen: Niemeyer.

Sommerfeldt, K. E. / Schreiber, H. (1977/¹983). Wörterbuch zur Valenz und Distribution deutscher Substantive. Tübingen: Niemeyer.

Sommerfeldt, K. E. / Schreiber, H. (1996). Wörterbuch der Valenz etymologisch verwandter Wörter. Tübingen: Niemeyer.

Valbu $\rightarrow$ Schumacher, H. et alii.

Wahrig (2008). Großwörterbuch Deutsch als Fremdsprache. Berlin: Cornelsen. $\rightarrow W$-DaF.

W-DAF: $\rightarrow$ Wahrig. Großwörterbuch Deutsch als Fremdsprache.

Wehrle H. / Eggers, H. (1961). Deutscher Wortschatz. Stuttgart: Klett.

\section{Artículos y monografías}

Alonso Ramos, M. (2004). «Elaboración del Diccionario de colocaciones del español y sus aplicaciones». En: P. Battaner Arias y J. De Cesaris (eds.). De Lexicografía. (Actes del I Simposium Internacional de Lexicografía) Barcelona. IULA y Edicions Petició: 149-162.

Bernal, E. / Renau, I. (2010). «¿Lo que necesitan es lo que encuentran? Reflexiones a propósito de la representación de los verbos en los diccionarios de aprendizaje del español». En: A. Dins Dykstra / T. Schoonheim (eds.). Proceedings of the XIV Euralex International Congress (Leeuwarden, 6-10 July 2010). Ljouwert: Fryske Akademy. 484-496.

Boas, H. C. / Dux, R. (2013). «Semantic frames for foreign-language education: Towards a German frame-based dictionary». Veridas On-Line 1/2013: 81100.

Bubenhofer, N. / Ptashnyk, St. (eds.) (2010). Korpora, Web und Datenbanken: Computergestützte Methoden in der modernen Phraseologie und Lexikographie. (Phraseologie und Parömiologie, 25). Nürnberg: Schneider Verlag Hohengehren.

Coseriu, E. (1977/v. esp. $\left.{ }^{2} 1986\right)$. Principios de semántica estructural. Madrid: Gredos. 
Cosma, R. et alii (eds.) (2014). Komplexe Prädikationen als Argumente. Kontrastive Untersuchungen zum Deutschen, Rumänischen und Englischen. Berlin. Akademie-Verlag.

Domínguez Vázquez, Maa J. et alii (2013). «Wörterbuchbenutzung: Erwartungen und Bedürfnisse. Ergebnisse einer Umfrage bei Deutsch lernenden Hispanophonen». En: Ma J. Domínguez VÁzquez (ed.). Trends in der deutschspanischen Lexikographie. Frankfurt: P. Lang Edition: 135-172.

Egido Vicente, Mª (2015). «Das konzeptuelle Feld der AUTORITÄTSBEZIEHUNGEN aus kontrastiver Sicht. Eine korpusbasierte Studie des Deutschen und Spanischen». En: St. Engelberg et alii (eds.). Argumentstruktur zwischen Valenz und Konstruktion. (= Studien zur deutschen Sprache, 68). Tübingen: G. Narr Verlag: 365-377.

Egido Vicente, Ma ${ }^{a}$ et alii (2015). «A new pedagogical dictionary for DaF and Ele with an onomasiological focus». En: J. P. Silvestre y A. Villaldva (eds.). Planning non-existent dictionaries. Lisboa. Centro de Linguística da Universidade de Lisboa / Universidade de Aveiro: 143-151.

Engel, U. (2004). Deutsche Grammatik - Neubearbeitung. München: iudicium.

ENGELBERG, St. (2015a). «The argument structure of psych-verbs: A quantitative corpus study on cognitive entrenchment». En: H. BoAs y A. Ziem (eds.). Constructional approaches to argument structure in German. Boston / Berlin: De Gruyter Mouton. (en prensa).

Engelberg, St. (2015b). «Gespaltene Stimulus-Argumente bei Psych-Verben. Quantitative Verteilungsdaten als Indikator für die Dynamik sprachlichen Wissens über Argumentstrukturen». En: St. Engelberg et alii (eds.). Argumentstruktur zwischen Valenz und Konstruktion. (= Studien zur deutschen Sprache, 68). Tübingen: G. Narr: 469-493.

Engelberg, St. et alii (2012). «Argument structure and text genre: crosscorpus evaluation of the distributional characteristics of argument structure realizations». Lexicographica 28: 13-48.

Engelberg, St. / Lemnitzer, L. (2001/ ${ }^{42009) . ~ L e x i k o g r a p h i e ~ u n d ~ W o ̈ r t e r b u c h-~}$ benutzung. Tübingen: Stauffenburg.

Fernández Méndez, M. (2014). «¿irve para algo un diccionario monolingüe de ELE?». En: Ma. P. Garcés Gómez (eds.). Lexicografía Teórica y aplicada, Anexos de Revista de Lexicografía, 26. A Coruña: Universidade de A Coruña, Servicio de Publicacións: 153-164.

- (2015). «Argumentstrukturmuster und valenzgrammatisch orientierte Information im deutsch-spanischen Kontrast: exemplarische Analyse der Lexikalisierungsmöglichkeiten des Konzepts ENTFÜHREN». En: ST. ENGEL- 
Berg et alii (eds.). Argumentstruktur zwischen Valenz und Konstruktion. (= Studien zur deutschen Sprache 68). Tübingen: G. Narr: 353-365.

Fernández Méndez, M. et alii (2015). «DICONALE-online: propuesta de un nuevo tipo de diccionario pedagógico bilingüe alemán-español para la producción en lengua extranjera». En: M. Meliss et alii (eds.) (2015). La lexicografía románica del siglo XxI. München. iudicium. (en prensa).

- (2016). «Sobre el uso de diccionarios para Ele y DaF: Análisis de una encuesta desde una perspectiva contrastiva». En: F. Robles I SAbater et alii (eds.). Tübingen: G. Narr (= Romanistische Fremdsprachenforschung und Unterrichtsentwicklung). (en prensa).

Fuentes Morán, Ma Teresa (1997). Gramática en la lexicografía bilingüe. Morfología y sintaxis en diccionarios español-alemán desde el punto de vista del germanobablante. Tübingen: Niemeyer.

García-Miguel, J. M. et alii (2010). «ADESSE. A Database with Syntactic and Semantic Annotation of a Corpus of Spanish». En: N. Calzolari et alii (eds.). Proceedings of the Seventh International Conference on Language Resources and Evaluation (LREC) (Valletta-Malta, 17-23 de mayo): 1903-1910. [<http://www.lrec-conf.org/proceedings/lrec2010/index.html>].

Geckeler, H. (1971/31982). Strukturelle Semantik und Wortfeldtheorie. München: W. Fink.

González Ribao, V. (2015a). «Sobre algunos conflictos en la 'pre'-lexicografía: la selección de corpus para la elaboración de un diccionario contrastivo alemán-español». Enः Mª J. Domínguez VÁzquez et alii (eds.). Lexicografía de las lenguas románicas. A proximaciones a la lexicografía moderna y contrastiva. (Coords.: Ma D. Sánchez Palomino y Ma J. Domínguez Vázquez), (vol. 2). Berlin/München/Bosten: De Gruyter. 247-269.

González Ribao, V. (2015b). «Das lexikosemantische Paradigma der neuen medialen Kommunikationsverben im Sprachvergleich (Spanisch-EnglischDeutsch) unter dem Blickwinkel der Argumentstruktur». En: St. EngelBERG et alii (eds.). Argumentstruktur zwischen Valenz und Konstruktion. (= Studien zur deutschen Sprache, 68). Tübingen: G. Narr: 341-353.

González Ribao, V./ Meliss, Meike (2015). «Theoretische und methodologische Grundlagen eines onomasiologisch-konzeptuell orientierten Produktionswörterbuches für den zweisprachigen Lernerkontext: Deutsch-Spanisch». En: J. A. Calañas Continente y F. Robles i Sabater (eds.). Los diccionarios de alemán: evolución y nuevas perspectivas. Frankfurt. P. Lang (= Spanische Akzente. Studien zur Linguistik des Deutschen, 2): 109-136. 
González Ribao, V. / Proost, K. (2015). «El campo léxico al servicio de la lexicografía: Un análisis contrastivo en torno a algunos subcampos de los verbos de comunicación en alemán y español». Enः Ma J. Domínguez VÁzquez et alii (eds.). Lexicografía de las lenguas románicas. Aproximaciones a la lexicografía moderna y contrastiva. (Coords.: Ma D. SÁnchez Palomino y Ma J. Domínguez Vázquez), (vol. 2). Berlin/München/Bosten: De Gruyter: 223-245.

Haensch, G. / Omeñaca, C. (1997, 2004). Los diccionarios del español en el siglo XXI. Salamanca: Ediciones Universidad.

Hass-Zumкenr, U. (2001). Deutsche Wörterbücher. Berlin / New York: De Gruyter.

Hausmann, F. J. (1991). «Die zweisprachige Lexikographie Spanisch-Deutsch, Deutsch-Spanisch». En: F. J. HAusmann et alii (1989-1991). Wörterbücher: Ein Internationales Handbuch zur Lexikographie. HSK 5.1-5.3, Berlin / NewYork: De Gruyter $2987-2991$.

Lemnitzer, L. / Zinsmeister, H. (2006). Korpuslinguistik. Eine Einfübrung. Tübingen: Narr.

Martín Mingorance, L. (1994). «La lexicografía onomasiológica». En: H. Hernández / H. Mederos (Coord.). Aspectos de lexicografía contemporánea., Barcelona. Biblograf: 15-27.

Meliss, M. (2005). Recursos lingüisticos alemanes relativos a «GER ÄUSCH» y sus posibles correspondencias en español. Un estudio lexicológico modular-integrativo. Frankfurt: P. Lang.

- (2013a). «Das zweisprachige Wörterbuch im bilateralen deutsch-spanischen Kontext. Alte und neue Wege». En: Ma J. Domínguez Vázquez (ed.). Trends in der deutsch-spanischen Lexikographie. Frankfurt: P. Lang Edition: 61-87.

Meliss, M. (2013b). «Online-Lexikographie im DaF-Bereich: Eine erste kritische Annäherung: Bestandsaufnahme - Nutzen - Perspektiven». Real: Revista de Estudos Alemães, 4/2013: 176-199 [<http://real.fl.ul.pt/textos.page/ $\operatorname{pag} / 2>]$.

- (2014a). «(Vor)überlegungen zu einem zweisprachigen Produktionslernerwörterbuch für das Sprachenpaar DaF und ELE». En: D. ReimanN (ed.). Kontrastive Linguistik und Fremdsprachendidaktik Iberoromanisch Deutsch. Studien zu Morphosyntax, nonverbaler Kommunikation, Mediensprache, Lexikographie und Mehrsprachigkeitsdidaktik (Spanisch/Portugiesisch/ Deutsch) (= Romanistische Fremdsprachenforschung und Unterrichtsentwicklung, 2). Tübingen. Narr: 113-137. 
- (2014b). «Die fremdsprachige Produktionssituation im Fokus eines onomasiologisch-konzeptuell orientierten, zweisprachig-bilateralen Wörterbuches für das Sprachenpaar Deutsch - Spanisch: Theoretische und methodologische Grundlagen von DICONALE». En: A. Abel et alii (eds.) (2014). Proceedings of the XVI EURALEX International Congress: The User in Focus. Bolzano: Institute for Specialised Communication and Multilingualism: 1119-1134. [<http:// www.euralex.org/elx_proceedings/Euralex2014/euralex_2014_087p_1119. pdf $>$.

- (2015a). «Propuestas para un diccionario conceptual bilingüe para Ele y DaF: ¿Qué aportan los diccionarios de aprendizaje para situaciones de expresión escrita en lengua extranjera?». En: Ma J. Domínguez Vázquez et alii (eds.). Lexicografía de las lenguas románicas. Aproximaciones a la lexicografía moderna y contrastiva. (Coords.: Ma D. SÁnchez Palomino y Ma J. Domínguez VÁzquez), (vol. 2). Berlin/München/Bosten: De Gruyter: 271-298.

- (2015b). «Das verbale Kombinationspotenzial in einsprachigen DaF-Lernerwörterbüchern: Kritische Bestandsaufnahme - Neue Anforderungen». Deutsch als Fremdsprache. Zeitschrift zur Theorie und Praxis des Faches Deutsch als Fremdsprache. Berlin: E. Schmidt. ZDaF, 1/2015: 14-27.

- (2015c). «Argumentstrukturen, Valenz und Konstruktionen: Eine korpusbasierte Studie deutscher und spanischer,Geruchsverben ' im Kontrast». En: ST. Engelberg et alii (eds). Argumentstruktur zwischen Valenz und Konstruktion. (= Studien zur deutschen Sprache 68). Tübingen: G. Narr: 317-341.

— (2015d). «Was suchen und finden Lerner des Deutschen als Fremdsprache in aktuellen Wörterbüchern? Auswertung einer Umfrage und Anforderungen an eine aktuelle DaF-Lernerlexikographie». En: Th. Roelcke (ed.). Wörterbücher für Deutsch als Fremdsprache - Probleme und Perspektiven. Info/DaF (en prensa).

Meliss, M. (2015e). «Wie viele und welche bilingualen Online-Wörterbücher brauchen wir für den DaF-Bereich? Erstellung eines Kriterienrasters und erste Bestandsaufnahme aus der Sicht eines hispanophonen Lernerkontextes». En: J. Cuartero Otal et alii (eds.) Querschnitt durch die deutsche SprachePerspektiven der Kontrastiven Linguistik. Berlin: Frank \&Timme (en prensa).

Meliss, M. / Sánchez Hernández, P. (2016). «La perspectiva conceptual onomasiológica en la lexicografía de aprendizaje del español como lengua extranjera: nuevos impulsos». En: $M^{a}$ D. Sánchez Palomino et alii (eds.) (2016). Lexicografía iberorománica. (en preparación).

Model, Benedikt (2010). Syntagmatik im zweisprachigen Wörterbuch. Berlin: De Gruyter. 
Müller-Spitzer, C. (ed.) (2014). Using Online Dictionaries. Berlin / Boston: De Gruyter (Lexicographica: Series Maior 145).

Porto Dapena, J. Á. (2002). Manual de técnica lexicográfica. Madrid: Arco.

Porto Dapena, J. Á. et alii (2008). «Presentación del diccionario 'Coruña' de la lengua española actual». Enः E. Bernal y J. DeCesaris (eds.). Proceedings of the Xiii Euralex International Congress. Barcelona. Documenta Universitaria (Série Activitats, 20): 753-762.

Reichmann, O. (1989). «Das onomasiologische Wörterbuch: Ein Überblick». En: F. J. Hausmann et alii (1989-1991). Wörterbücher: Ein Internationales Handbuch zur Lexikographie. HSK 5.1-5.3, Berlin / NewYork: De Gruyter: 1057-1067.

Renau, I. / Battaner, P. (2012). «Respuesta a la necesidad de diccionarios en línea para el aprendizaje de español como lengua extranjera». En: J. ACEBRón Ruiz et alii (eds.). Tonto el que no lea. Enseñar lengua y literatura castellanas en la era digital. Lleida: Universidad de Lleida: 149-158.

Renouf, A. (ed.) (2009). Corpus linguistics: refinements and reassessments. Amsterdam: [u.a.] Rodopi (Language and computers, 69).

Sánchez Hernández, P. (2010). «Análisis contrastivo alemán español de los verbos fragen-antworten/ lehren-lernen». Revista de Filología Alemana, Madrid, Servicio de Publicaciones UCM, 18/2010ः 261-283.

SÁnchez Hernández, P. (2012) + «'Lernen-aprender’ ‘ una aproximación contrastiva dentro del campo semántico Kognition en torno a diversas peculiaridades semánticas y sintácticas». Revista de Filología alemana, Madrid, Servicio de $\mathrm{Pu}$ blicaciones UCM, 20/2012: 139-158.

Sánchez Hernández, P. (2013a)+ «La lematización de las unidades fraseológicas en diccionarios generales semasiológicos y onomasiológicos». Enः C. MELLADO (coord.) et alii (eds.). - La fraseología del alemán y el español: lexicografía y traducción. (Reihe: Linguistische Studien). Münchenः Peniope: 129-143.

- (2013b). «Zur Konzipierung eines deutsch-spanischen kombiniert onomasiologisch-semasiologisch ausgerichteten Verbwörterbuchs mit online-Zugriff - ausgewählte Aspekte». Aussiger Beiträge, Ustí nad Labem, Praesens, 7/2013: 135-155.

Sánchez Hernández, P. (2015). «Überlegungen zu der syntagmatischen Information einiger Verben des Teilparadigmas LERNEN im deutschspanischen Vergleich». En* St. Engelberg et alii (eds.). Argumentstruktur zwischen Valenz und Konstruktion. (= Studien zur deutschen Sprache, 68). Tübingen: G. Narr: 377-394. 
Subirats, C. (2009). «Spanish Framenet: A frame-semantic analysis of the Spanish lexicon». En: H. BoAs (ed.). Multilingual FrameNets in Computational Lexicography. Methods and Applications. Berlin / New York: Mouton de Gruyter: 135-162.

TARp, S. (2012). «Online dictionaries: today and tomorrow». En: U. Heid (ed.). Thematic Part: Corpora and Lexicography. Berlin: de Gruyter. Lexicographica 28/2012: 253-267.

Trujillo, R. (1970). El campo semántico de la valoración intelectual en español. La Laguna: Universidad de La Laguna.

Werner, R. (1998). «La selección de lemas en los diccionarios español-alemán y alemán-español o çun diccionario de qué lengua es un diccionario de las lenguas española y alemana?». En: Ma T. Fuentes Morán y R. Werner (eds.). Lexicografías iberorománicas: problemas, propuestas y proyectos. Frankfurt a. M.: Vervuert: 139-156.

Wotjak, G. (2013). «Inwieweit kann das kommunikative Potenzial lexikalischer Einheiten als Bezugsbasis für kontrastive Lexikographie im Sprachenpaar Spanisch-Deutsch dienen?» En: Ma J. Domínguez Vázquez (ed.). Trends in der deutsch-spanischen Lexikographie. Frankfurt: P. Lang Edition: 109-133. 